\title{
UN DISEÑO DE “MEDIDA Y DIAPASÓN”, PATRÓN PARA UN ÓRGANO DE LA CATEDRAL DE TOLEDO DE 1584*
}

\section{A NEW PATTERN FOR ORGAN MUSIC: THE DESIGN OF "MEASURE AND TUNE' FOR AN ORGAN IN TOLEDO'S CATHEDRAL OF 1584}

\author{
Louis Jambou \\ Universidad de París IV-Sorbonne
}

\begin{abstract}
Resumen
El nuevo equipo directivo del archivo de la Catedral de Toledo ha puesto en marcha un programa de ediciones que van a permitir mejor apreciar el repertorio de la antigua capilla de música. Recientemente se ha descubierto el diseño de un diapasón de caño de órgano que sirvió para la hechura de un órgano de 1584. El artículo es una primera descripción y estudio de esta herrmanienta en algunos de sus parámetros así como en su contexto toledano, español o internacional. Un estudio acústico más preciso acercará a los músicos de la realidad interpretativa de la música de la segunda mitad del siglo XVI.
\end{abstract}

\section{Palabras clave}

España, Toledo, Organería siglo XVI, Diapasón, Medidas interválicas, Altura del sonido, Acústica.

Desde 2007, bajo la dirección e impulso de D. Ángel Fernández Collado y su equipo de técnicos archiveros, Isidoro Castañeda Tordera y Alfredo Rodríguez González, el archivo de la catedral primada de Toledo ha tomado un nuevo rumbo de actividades y de publicaciones entre las cuales cabe destacar Los diseños de la catedral de Tole$d o^{1}$. Este volumen contiene, entre otros diseños, los dibujos

\footnotetext{
* Nuestro más vivo agradecimiento al Director de Anuario Musical, Luis Antonio González Marín, por acoger, en un número ya cerrado dedicado a la obra de Cabezón, esta crónica anunciando este hallazgo.

1 FERNÁNDEZ COLLADO, RODRÍGUEZ GONZÁLEZ y CASTAÑEDA TORDERA, 2009.
}

\begin{abstract}
Thanks to an editorial program in process in Toledo's cathedral our knowledge of the musical repertoire intended for the musical chapel in Toledo will be better known and understood. The recent discover of a design of a pitch pipe for a 1584 organ used in Toledo's cathedral sheds new Light on tuning issues during the Renaissance. This article's aim is to offer a preliminary report of this design, placing it within tune parameters of the time as well as setting it in both Toledan and international contexts. Accurate future acoustics research on this design will probably allow us to have a better knowledge of performances pratices during the second half of the $16^{\text {th }}$ century.
\end{abstract}

\section{Key words}

Spain, Toledo, Organ builders in the $16^{\text {th }}$ century, Tune, Intervals measures, Pitch, Acoustics.

preparatorios para la construcción de cajas de órganos. La musicología no queda apartada de estas preocupaciones ya que el centro editorial promete la pronta salida de valiosos trabajos de Michael Noone y de Carlos Martínez Gil. Ahora el equipo técnico nos ofrece ${ }^{2}$ un diseño del siglo XVI que es

2 Lo encontramos encima de la mesa de trabajo: lo consultamos el 30 de enero del 2014 y los días siguientes coincidiendo con la celebración del simposio "El entorno musical del Greco" (30/0102/02/2014). Agradecemos a los archiveros y al cabildo catedralicio su amabilidad en proporcionarnos esta gran sorpresa. La emoción fue grande al desplegarlo y descubrir su contenido. También pudimos consultar una caja de documentos sobre los "Órganos" que conserva copias de los documentos notariales (desde el siglo XVI) de la fábrica o sobre 
un "diapasón" de un caño de órgano y de su registro. En la musicología este diapasón, molde y patrón para la acción, es un gran hallazgo tanto por sus indicaciones como por el corto o nulo número de su presencia en los fondos o museos musicales $^{3}$. La descripción que sigue será una mera presentación y no agotará las reflexiones que sugiere ; éstas, aquí, reflejarán unos esbozos analíticos, una contextualización temporal y geográfica inmediata que se empezará a ampliar pero esperará otras contribuciones.

\section{PRESENTACIÓN}

El dibujo se presenta en una tira o cinta de papel cuadrangular doblada en 7 pliegues con refuerzos pegadas en las dobleces y formando 8 segmentos. El papel es de fuerte textura textil, con media marca de agua en el borde del anverso. El largo de cada pliego es de $19 \mathrm{cms}$ con un ancho constante de $9 \mathrm{cms}$ a lo largo de la cinta. El largo total de ésta es de 155/156 cms. Se lee en forma vertical y no horizontal : el diseño es la reproducción de un tubo.

En el anverso de la cinta viene escrito con letra del siglo XVI "medida y diapason del horgano q[ue] se ha de hazer para la prosizion [sic] de corpus criste de la sancta yglesia"4.
En el recto, a nivel de la raya del Fa4, se lee " $o$ [ $¿$ ?], $a$ este alto", dejando suponer que se vaciló en la altura definitiva que se le quería dar al tubo más largo.

El diseño (recto o anverso) no tiene firma ni fecha.

En el recto viene el diseño.

Siendo el largo total de la cinta de 155/156 $\mathrm{cms}^{5}$ el dibujo de la cara recto presenta dos partes : el pie del caño y el caño sonante propiamente dicho.

El largo total del caño con el pie (de punto o raya horizontal superior al mismo punto o raya inferior : $152 \mathrm{cms}$ $+\mathrm{o} \mathrm{cms}$

El alto del pie hasta la base de la boca : $19 \mathrm{cms} 50$.

El alto del pie hasta lo alto de la boca : $21 \mathrm{cms} 50$.

El alto de la boca : $2 \mathrm{cms}$

El largo del caño 'sin el pie' ${ }^{66}$ ( se mide a partir del labio inferior de la boca). El caño dibujado representa todos los caños de un registro; mide $133 \mathrm{cms}$. Cada uno de los caños tiene un trazo horizontal y trazado medio círculo (a partir del sol2) que indica el grosor del tubo (su diámetro o talla) y permitiría así acercarnos a su realización. La altura de la tubería, lo único que se ha medido hasta ahora y lo único cuyo estudio se esboza aquí, se desglosa como sigue:

5 Los pliegues del papel impiden tener una medida exacta. Las medidas de loas caños se dan en centímetros sin su tranformación en "cent", medida de la acústica moderna.

$6 \mathrm{El}$ pie tiene el largo que se quiera ya que no sirve sino tan solo para llevar el viento hasta la lengueta repite Mersenne varias veces [cito y traduzco de memoria]. 
1) Medidas de la cañería o tubería ${ }^{7}$

\begin{tabular}{|c|c|c|c|}
\hline Primer diapasón u octava & Segundo diapasón u octava & Tercer diapasón u octava & Cuarto diapasón u octava \\
\hline \multirow{2}{*}{ 1-. Do1=133 cm } & 9. Do2 $=67 \mathrm{cms} 4$ & 21.- Do3=33cms9 & 33. Do4= 16cms9 \\
\hline & 10. Do2\#=64cms & 22. Do\#3=32 cms & 34. Do4\#=16 cms \\
\hline \multirow[t]{2}{*}{ 2. $\operatorname{Re} 1=119 \mathrm{cms} 5$} & 11. $\operatorname{Re} 2=\mathbf{6 0} \mathbf{~ c m s}$ & 23. $\operatorname{Re} 3=\mathbf{3 0} \mathbf{c m s}$ & 35. $\operatorname{Re} 4=15 \mathrm{cms}$ \\
\hline & 12. $\mathrm{Mi} 2 \mathrm{~b}=\mathbf{5 7} \mathrm{cms}$ & 24. Mib3=28cms4 & 36. Mib4=14cms 2 \\
\hline 3. Mi1 $=107 \mathrm{cms} 5$ & 13. $\mathrm{Mi} 2=\mathbf{5 3} \mathbf{c m s} \mathbf{2}$ & 25. Mi3=26cms6 & 37. $\mathrm{Mi} 4=\mathbf{1 3} \mathbf{c m s 3}$ \\
\hline \multirow[t]{2}{*}{ 4. $\mathrm{Fa} 1=101 \mathrm{cms} 3$} & 14. $\mathrm{Fa} 2=\mathbf{5 0} \mathrm{cms} 7$ & 26. $\mathrm{Fa}=\mathbf{2 5} \mathrm{cms} 2$ & 38. Fa4= 12cms5 \\
\hline & 15-. Fa2\#=47cms5 & 27. $\mathrm{Fa} 3 \#=\mathbf{2 3} \mathbf{c m s 8}$ & 39. Fa4\#=11 cms8 \\
\hline \multirow[t]{2}{*}{ 5-. Sol1= $90 \mathrm{cms}$} & 16-. Sol2=44cms9 & 28. Sol3=22cms 5 & 40. Sol4=11cms1 \\
\hline & 17. Sol2\#=42cms6 & 29. Sol3\#= 21cms 4 & 41. Sol4\#= 10cms6 \\
\hline 6. La1=80cms7 & 18. La2 =39cms9 & 30. $\mathrm{La} 3=\mathbf{2 0} \mathbf{c m s}$ & \\
\hline 7-. $\mathrm{Sib1} 1=\mathbf{7 6} \mathrm{cms}$ & 19. Sib2 $=\mathbf{3 7} \mathbf{c m s 9}$ & 31. Sib3=18cms8 & \\
\hline 8. $\mathrm{Si} 1=71 \mathrm{cms} 3$ & 20. $\mathrm{Si} 2=\mathbf{3 5} \mathbf{c m s} \mathbf{8}$ & 32. $\mathrm{Si} 3=\mathbf{1 7} \mathbf{c m s 9}$ & \\
\hline
\end{tabular}

2) Hechas las medidas interválicas cromáticas desglosadas intermediarias llegamos al cuadro final de medidas interválicas cromáticas y diatónicas que sigue.

\begin{tabular}{|c|c|c|c|}
\hline Primer diapasón u octava & Segundo diapasón u octava & Tercer diapasón u octava & Cuarto diapasón u octava \\
\hline \multirow[t]{3}{*}{ 1. Do1-Re1=13,5 } & 9. Do2-Do2\#=3,4 & 21. Do3-Do3\#= 1,9 & 33. Do4-Do\#4=0,9 \\
\hline & 10. $\mathrm{Do} \# 2-\mathrm{Re}=\mathbf{4 , 0 0}$ & 22. Do\#3-Re3=2,00 & 34. Do\#4-Re4= 1,00 \\
\hline & $10^{\circ}$. Do2-Re2=7.4 & 22'. Do3-Re3=3,9 & $34^{\circ}$. Do4-Re4=1,9 \\
\hline \multirow[t]{3}{*}{ 2. Re1-Mi1=12.00 } & 11. $\operatorname{Re} 2-M i b 2=\mathbf{3 , 0 0}$ & 23. Re3-Mib3=1,6 & 35. Re4-Mib4=0,8 \\
\hline & 12. $\mathrm{Mi} 2 \mathrm{~b}-\mathrm{Mi} 2=\mathbf{3 , 8}$ & 24. Mib3-MI=1,8 & 36-. Mib4-Mi4=0,9 \\
\hline & $12^{`} \cdot \operatorname{Re} 2-\mathrm{Mi} 2=\mathbf{6 , 8}$ & $24^{\prime} . \mathrm{Re} 3-\mathrm{Mi} 3=\mathbf{3 . 4}$ & $36^{\circ} . \mathrm{Re} 4-\mathrm{Mi} 4=\mathbf{1 , 7}$ \\
\hline 3. $\mathrm{Mi} 1-\mathrm{Fa} 1=6,2$ & 13. $\mathrm{Mi} 2-\mathrm{Fa} 2=\mathbf{2 , 5}$ & 25. $\mathrm{Mi} 3-\mathrm{Fa} 3=\mathbf{1 , 4}$ & 37, Mi4-Fa4= $\mathbf{0 , 8}$ \\
\hline \multirow[t]{3}{*}{ 4. Fa1-Sol1=11,3 } & 14. $\mathrm{Fa} 2-\mathrm{Fa} 2 \#=\mathbf{3 , 2}$ & 26. $\mathrm{Fa} 3-\mathrm{Fa} \#=1.4$ & 38. $\mathrm{Fa} 4-\mathrm{Fa} 4 \#=\mathbf{0 , 7}$ \\
\hline & 15. Fa2\#-Sol2=2,6 & 27. Fa\#3-Sol3=1,3 & 39- Fa4\#-Sol4=0,7 \\
\hline & $15^{`} . \mathrm{Fa} 2-\mathrm{Sol} 2=\mathbf{5 , 8}$ & $27^{`} . \mathrm{Fa} 3-\mathrm{Sol} 3=\mathbf{2 , 7}$ & $39^{‘}$. Fa4-Sol4=1,4 \\
\hline \multirow[t]{2}{*}{ 5. Sol1-La1=9,3 } & 16. Sol2-Sol2\#=2,3 & 28-.Sol3-Sol\#3=1,1 & 40. Sol4-Sol\#4=0,5 \\
\hline & 17. Sol2\#-La2=2,7 & 29. Sol\#3-La3= $\mathbf{1 , 4}$ & 41. Sol\#4 \\
\hline 6. La1-Sib1= 4,7 & 18. La2-Sib=2,00 & 30. La3-Sib3= 1,2 & \\
\hline $6^{6}$. La1- Si1 $=\mathbf{8 , 4}$ & 18‘. La2-Si2=4,1 & 30'. La3-Si3=2,1 & \\
\hline 7. Sib1-Do2 =8,6 & 19. $\mathrm{Sib} 2-\mathrm{Si}=\mathbf{2 , \mathbf { 1 }}$ & 31-. Sib3-Si=1 0,9 & \\
\hline 8. Si1-Do2= $\mathbf{3 , 9}$ & 20-. Si2-Do3=1,9 & 32-. Si3-Do4=1,00 & \\
\hline $8^{6} \cdot \mathrm{Si} 1=\mathrm{Do} \# 2=\mathbf{7 , 3}$ & $20^{\circ} . \mathrm{Si} 2-\mathrm{Do} \# 3=\mathbf{3 . 8}$ & 32‘. Si3-Do\#4=1,9 & \\
\hline
\end{tabular}

7 Agradecemos a Juan José Montero, director del Conservatorio “Jacinto Guerrero" de Toledo, el proporcionarnos estas medidas. Organista y experto en órganos Juan José Montero ha trabajado en el taller toledano de Gerard A. C. De Graaf durante unos diez años. 


\section{PRIMERAS OBSERVACIONES}

En 1) -

el teclado así dibujado es un teclado basado en $U t$ y de octava corta ; es decir que no tiene los accidentales sostenidos de la primera octava de $U t$, Sol ni el bemol de $M i$. Abarca un ámbito de 41 teclas de $U t^{1}$ a Sol${ }^{4} \#$. Se fundamenta en el $U t^{1}$ pero no llega al $\mathrm{La}^{4}$, es decir que no llega al ámbito más habitual de la época de 42 teclas de $U t^{1}$ a $L a^{4}$.
-El tubo mayor tiene un alto de $133 \mathrm{cms}$ : corresponde a un tubo de 6 palmos y medio; pero se ha de notar que ha de hacerse "en tono de chirimía" según puntualiza el contrato (véase más abajo).

Entre los semitonos interválicos diatónicos (mi-fa y sido) no prevalece la estabilidad que se podría esperar :
3-Mi1-Fa1=6,2 13- $\mathrm{Mi} 2-\mathrm{Fa} 2=\mathbf{2 , 5}$
25- Mi3-Fa3=1,4

37- Mi4-Fa4= 0,8
32-. Si3-Do4=1,00

-quedan manifiestas las medidas irregulares de los intervalos que no parecen seguir una proporción simple como la dupla que sería lógico de una octava a otra. Lo que se da sin embargo en los semitonos accidentales :

\begin{tabular}{|l|l|l}
\hline $10-\mathrm{Do} \# 2-\mathrm{Re}=\mathbf{4}$ & 22-Do\#3-Re3=2,00 & 34- Do\#4-Re4= 1,00
\end{tabular}

y en las tres octavas superiores cuya primera octava queda alta (12cms)

\begin{tabular}{|l|l|l|}
\hline $12^{6}-\operatorname{Re} 2-\mathrm{Mi} 2=\mathbf{6 , 8}$ & $24^{6}-\mathrm{Re} 3-\mathrm{Mi} 3=\mathbf{3 . 4}$ & $36^{6}-\mathrm{Re} 4-\mathrm{Mi} 4=\mathbf{1 , 7}$ \\
\hline
\end{tabular}

o en dos octavas finales :

\section{3- Re3-Mib3= 1,6}

24- Mib3-MI=1,8

u en otras

En 2)

el temperamento no es un temperamento de semitonos iguales, como podría ser el caso de la cuerda pulasada, como se nota entre las distintas notas accidentales y sus octavas :

\begin{tabular}{|c|c|c|}
\hline 9- Do2-Do2\#=3,4 & 21-Do3-Do3\#= 1,9 & 33- Do4-Do\#4=0,9 \\
\hline $10-\mathrm{Do} \# 2-\mathrm{Re}=4$ & 22-Do\#3-Re3=2,00 & 34- Do\#4-Re4= 1,00 \\
\hline
\end{tabular}

\begin{tabular}{|l|l|l|}
\hline 11-Re2-Mib2=3,00 & 23- Re3-Mib3= 1,6 & 35- Re4-Mib4=0,8 \\
\hline 12- Mi2b-Mi2=3,8 & 24- Mib3-MI=1,8 & 36- Mib4-Mi4=0,9 \\
\hline
\end{tabular}

\begin{tabular}{|l|l|l|}
\hline $14-\mathrm{Fa} 2-\mathrm{Fa} 2 \#=\mathbf{3 , 2}$ & $26-\mathrm{Fa} 3-\mathrm{Fa} \#=\mathbf{1 . 4}$ & $38-\mathrm{Fa} 4-\mathrm{Fa} 4 \#=\mathbf{0 , 7}$ \\
\hline $15-\mathrm{Fa} 2 \#-\mathrm{Sol} 2=\mathbf{2 , 6}$ & $27-\mathrm{Fa} \# 3-\mathrm{Sol} 3=\mathbf{1 , 3}$ & $39-\mathrm{Fa} 4 \#-\mathrm{Sol}=\mathbf{0 , 7}$ \\
\hline
\end{tabular}

\begin{tabular}{|c|c|c|}
\hline 6'- La1-Si1= 8,4 & $18^{`}-\mathrm{La} 2-\mathrm{Si} 2=\mathbf{4 , 1}$ & $30^{\circ}-\mathrm{La} 3-\mathrm{Si} 3=\mathbf{2 , 1}$ \\
\hline 7- Sib1-Do2 =8,6 & 19- $\mathrm{Sib} 2-\mathrm{Si}=\mathbf{2 , 1}$ & $31-\mathrm{Sib} 3-\mathrm{Si}=1 \mathbf{0 , 9}$ \\
\hline
\end{tabular}

En estas medidas se comprueban las oscilaciones que puede haber, y las discusiones que pueden suscitar y acarrear entre "semitono mayor" y "semitono menor", entre "semitono incantable" y "semitono cantable". 
Lo que significa que las medidas de este diapasón ${ }^{8}$ (salvo errores lo que siempre es posible) no tienen una altura absoluta sino que son relativas e indicativas de una práctica y corregibles o subsanables en la realización y afinación final del instrumento. Según palabras de Juan José Montero "nunca se plantea una medida exacta porque es necesario afinar después de construir el tubo, por lo que la medida es necesariamente distinta“ (Correo electrónico)

\section{SITUACIÓN CONTEXTUAL TEÓRICA EN ESPAÑA}

No cabe duda de que los teóricos del siglo XVI que dedican alguna atención a la hechura del órgano limitan sus observaciones a escasos capítulos ${ }^{9}$. Bermudo es el más valioso de ellos pero se queda en el umbral de lo que quisiéramos saber siendo una de sus obsesiones el instrumento "perfecto" de su momento, la vihuela, que a la fuerza ha de imitar el órgano, bien en su definición pitagórica bien en la de la división del tono en dos semitonos iguales. Se comprende así que su preocupación es la de una definición del diapasón, de su posibilidad o imposibilidad de la trasportación de todos los modos o tonos en cualquier punto de la octava. La conclusion a su libro IV sobre "tañer la vilhuela" puntualiza su anhelo más vivo:

"El que el sobredicho modo de preparar las sesquioctavas supiere exercitar en el hazer del organo: sacara el organo que se tanga[n] todos los semitonos. Y notese, que aunque son menester muchas cosas para que en el organo se tangan todos los semitonos: la potissima y principal es la sobredicha preparacion, de quitarle a los dos tono primeros la tercera parte de la co[m]ma. Y si la mitad de la co[m]ma le fuere quitada a la sobredicha tercera mayor quedaran mas sabrosas todas las terceras mayores, y sera tan pequeña la perdida de las quintas, que el oydo los juzgara por consonancias perfectas“"10.

Quedamos sin los libros finales del proyecto bermudiano de forma que no sabemos si llegó a concretar este an-

8 Véase, para el órgano español, la sintesis de los cap. 5 y 6 "Tono natural o de capilla" del libro de DE LA LAMA, 1995: 67-90.

9 JAMBOU,1988, vol. 1: 98-105, particularmente 99-100, la cita del "prólogo segundo" de Bermudo. revelan sus inventos deseados o realizados.

10 BERMUDO, 1555, L, IV, "De tañer vihuela“, fol. cix", cap. lxxxvi, "Dela vihuela de siepte ordenes que se tangan todos los semitonos" helo. ${ }^{11}$ A principios de siglo Juan de Espinosa ocupó varios cargos musicales en la catedral de Toledo. En esta capital imprime su "Tractado de prinicpios de música práctica y teórica sin deja ninguna cosa atras“ (1520). En éste la música instrumental es secundaria pero su modelo es el de la perfección instrumental, es el de los violeros "que hazen el mas perfecto instrumento que esta hecho ni se puede hazer"; embiste contra éstos lo mismo que arremete contra los "maestros de hacer organos" que

\begin{abstract}
"de muchos organistas que he p[r]ocurado de auer su diapason hallo entre ellos muy grandes diuersidades en las dichas cantidades: ansi en las de la orden inferior como en las de la superior y por esso nunca sacan organo ni es possible sacarle co[n] toda las perfectio[n] que ha menester de tener..."12.
\end{abstract}

Se entiende pues que en la música, dista aún el ser teórico del ser práctico en las realizaciones de los organeros. Observémoslas en lo que sabemos de los "maestros de hacer órganos" durante la segunda mitad del siglo.

\section{SITUACIÓN CONTEXTUAL PRÁCTICA TOLEDANA}

El libro arriba citado Los diseños de la catedral de Toledo presenta dibujos de cajas de órgano. Llaman la atención los proyectos presentados por Nicolás de Vergara el 9 de marzo de 1584 para la hechura de la caja de un "órgano de la proçesiones" ${ }^{\text {"13. }}$. Llama sobre todo la atención el texto que ilustra el diseño general (p.282-283) que es un borrador del que se presenta literalmente el mismo día, 3 de marzo de 1584, ante el escribano real Álvaro Pérez y que suscribe Nicolás de Vergara ${ }^{14}$. La misma escritura antepone las "condiciones del organo que se ha de hacer para las procesiones de la santa yglesia“" [de Toledo]. Estas condiciones declaran :

"Primeramente se ha de hacer un organo de seys palmos y medio tono de las chirimías. Porque asi se a tratado con el rracionero peraça y con jeronimo lopez.

11 Sobre este aspecto véase la tesis reciente de Andrés Cea Galán, La cifra hispana: Música, tañedores, instrumentos (siglos XVIXVIII), leída el 4 de junio de 2014 en la Universidad Complutense.

12 Cita entera en JAMBOU, 2007: 125-136. Por "orden inferior" u "orden superior" se ha de entender aquí, lo mismo que en Santa María, el rango de las teclas naturales, blancas, o acidentales, negras. Igulamente el vocablo "organista" es aquí sinónimo de "organero"

13 Libro citado nota 1: pp. 281-287 (n $\left.{ }^{\circ} 194\right)$

14 JAMBOU. 1988, vol. 2: 24-25, nº57 b. 
Tendra un flautado sonoroso y suabe [tachado : que tenga] doblado tendra un lleno muy sonoroso tendra en las contras cinco caños por punto y en los tiples siete con los del flautado toda esta cañuteria sera destaño fino sin mezcla de plomo estara asentada en un tablón de pino seco a la traça que diere bergara y quien hubiere de hacer la caja. Tendra un juego de buen toque de cuarenta y [tachado: dos] un puntos con sus tonos y semytonos [tachado : tendra]. Tendra dos fuelles de muy buena madera guarneçidos de baldreses doblados. [Escrito de otra letra: ] Yten que todo lo que pudiere aprovechar en este dicho organo de lo que esta en su poder que tenia que se lo entrego al dicho myranda se lo aproveche [vuelve a la primera letra :] todo lo qual hare en toda perfeçion a contento del rracionerio peraça y del señor obrero dentro de tres meses de como me dieren la caja y despues de echo se me tase y lo que mereçiere se me de por el . [Primera letra :] Guardense estas condiciones [Firman : ] Hieronimo López de Velasco. Hieronimo Peraza, Melchior de Myranda“ 15

En ellas se puntualiza pues que es un órgano de seis palmos y medio con "tono de las chrimías" lo que no deja de ser contradictorio con lo que se dirá más adelante. Melchor de Miranda es el organero oficial de la catedral desde 1581.

No queda duda : el dibujo encontrado en la Secretaría del Cabildo de Toledo, unido a la obligación notarial de Nicolás de Vergara de hacer la caja, tiene fecha y es de la mano del mismo Melchor de Miranda o de su obrador. Es de 1584 o anterior a esta fecha.

Se ha de descartar el nombre de Juan Bautista de Vargas como autor de este diseño, pese a que sea también autor de un órgano de procesiones hecho en 1572 ; curiosamente este instrumento se construye, además, según un diseño hecho por el mismo Nicolás de Vergara ${ }^{16}$. Pertenece a una familia de organeros, y es afinador titular de los órganos catedralicios entre 1566 y 1581, anterior pues a la titularidad de Melchor de Miranda. Pero ninguna cuenta o alusión permite hacerle autor del dibujo. En cambio la personalidad de Melchor de Vergara, su ascendencia y aliados y su propio linaje y su declive, dejan flotar alguna duda en la atribución del diseño. Formado en el taller de la dinastía más longeva de

15 No transcribimos aquí los términos del contrato de la caja que firma Nicolás de Vergara que se pueden leer en los libros citados.

16 El contrato (JAMBOU, 1988, vol. II: 10, n²2) precisa que es un órgano de procesiones del "Santísimo Sacramento que sale de la Santa Iglesia“. Es un detalle que tiene su importancia ya que deja suponer que era órgano portátil o realejo con un registro tapado y no abierto como el de 1584 . No se pueden sacar conclusions de hecho usual que dice el contrato que aproveche el material, de un órgano viejo sin duda, que le da el obrero o quien fuere.
Toledo, los Gómez, a partir de 1563, trabaja en su taller y se casa con una hija de su maestro Francisco I, María Felipa ; el matrimonio forma igualmente otra dinastía de organeros toledanos ${ }^{17}$.

La entonación del órgano merece también atención en relación con nuestros conocimientos. En Toledo, hasta el primer órgano conocido de Francisco Gómez I (1565), se construyen órganos de 12,13,14, 18 siendo los de 13 y 14 los más frecuentes. La variabilidad es pues marca del diapasón del instrumento. Entre los órganos de 6 palmos y $1 / 2$, se ha de notar, sobre todo, el de la iglesia parroquial de El Carpio (1551, por Juan Ximénez) que puntualiza que la entonación de 6 y $1 / 2$ ha de ser "muy apazible para oficiar el oficio divino", indicativo de su función de preludio y de acompañamiento.

Desde 1565 hasta ca. 1600 son 17 órganos los que se construyen de 13 palmos "sin el pie" ${ }^{\text {"18 }}, 15$ de los cuales son de los Gómez cuando los de 14 palmos llegan a 13 (de los Gómez o Miranda u otros) $)^{19}$. El "tono de chirimías" -en principio algo más alto que el tono natural o de capilla y que será luego el tono de orquesta- califica una vez el de 13 -el de este contrato - y otra vez el de $14^{20}$. Pero parece claro que la entonación del órgano toledano se orienta hacia la estabilización en 13 palmos $^{21}$. En ello parece seguir una evolución general que, en todo caso, se nota igualmente en Aragón donde en 1574 se declara que es "la entonación cantable que ahora se usa y se canta en todas las iglesias de Zaragoza“y que, en boca de Gaspar Marín formado en Toledo, se dice en 1588 que es "la mayor [=mejor] entonación para la capilla y de más perfección“22. Este superlativo aparece igualmente en contratos de Francisco Gómez “el Viejo“ o Francisco Gómez

17 Las obras de estos organeros pueden leerse en mi Evolución... y otros escritos. Se detallará en Les facteurs d'orgues en Espagne. 14001850 (en prensa, ed. Delatour-France)

18 Esta cláusula figura en muchos contratos pero no en todos; el pie del caño solía tener un palmo, un palmo y medio, o más en escasos casos (véase nota 6)

19 Es de notar que las otras entonaciones $(9,10,12$ y $1 / 2,13$ y $1 / 2$ $, 15,16,18)$ son de escaso número.

20 En Toledo este "tono de chirmías" sale en dos contratos de mano de Melchior de Miranda; una vez aquí en la catedral y otra , en 1580, en 1a iglesia del convento de San Francisco de Alcalá de Henares, (Jambou, Evolución..., , p. 17 n 42). Esta entonación queda algo imprecisa como se puede notar en estos dos ejmplos: uno es de 14 palmos y el otro, de la catedral, de 13 palmos. Ver también SAURA BUIL, 2001: 468. De La Lama lo cita "un punto más alto de lo natural" es casi exactamente como el "Cornetton alemán del s. s. XVIII (DE LA LAMA, 1995, vol I: 75 y 85).

21 En la nación vecina, Portugal, parece que el instrumento se ha orientado hacia una entonación en 12 palmos. Ver los ejemplos multiples, del siglo XVIII, en ESTEVES PEREIRA, 1984.

22 CALAHORRA MARTÍNEZ, 1977: 187-188 y 224-228. 
en 1580 y 1581 o $1583^{23}$. Nótese que esta entonación natural, de 13 palmos, no traza hacia una estabilización una línea tan recta como se pudiera creer $^{24}$.

En este contexto, toledano aquí, se ha de notar una herramienta "diapasón" que tiene que ver con lo que interesa este ensayo pero sobre cuya forma o soporte se tienen pocas noticias ${ }^{25}$. En el inventario de los bienes de Francisco Gómez, en 1589, aparecen "dos diapasones y una regla diez y seis reales" que pasan a su hijo Juan. En su testamento, en 1614, Juan Gómez declara que compró después de su segundo casamiento " $[\ldots]$ unas reglas $=$ unos diapasones $=[\ldots]$. $]$ "26. Durante estos años tal herramienta no aparece en los testamentos o inventarios de Juan Bautista de Vargas (1581), de Gaspar Brebos (1590), Andrés Gómez (1594) o Jerónimo de Vargas $(1598)^{27}$, aunque otros vocablos, como "molde", podrían tener el mismo significado.

23 JAMBOU, 1988: 16 (nº41, órgano para Almorox), 19 (n46, órgano para Lezuza), 22 ( $\mathrm{n}^{\circ} 22$, órgano para Cadahalso). Se ha de notar que estos contratos se hacen con el parecer y el dictado de Gerónimo Peraza elegido organista de la catedral en 1579.

24 Testimonio de ello son los distintos contratos en torno al órgano de la iglesia de Mondéjar que, por muerte de Francisco Gómez en 1589 , sufre varias etapas y coautores entre 1590 y 1694 , oscilando entre 13 (en 1590 con Andrés Gómez) y 14 palmos (en 1596 con Melchor de Miranda).

25 Recordamos haber leído, en el contexto aragonés o navarro, que desde algunos pueblos iban a la capital en busca de información sobre el diapasón en uso para la hechura del órgano del pueblo. No tenemos apuntes sobre este particular. Terminado ya este trabajo recibimos de Jesús Gonzálo López un correo electrónico (29/03/2014) puntualizando que en 1657 el organero francés Nicolás Brisset envió a la catedral de Tarazona tres tubos para comprobar la afinación del órgano de Fítero (Navarra) y que los músicos de la catedral le respondieron que los tubos eran "medio tono baxos" y entregaron a los portadores un tubo con la afinación justa (en SAGASETA ARIZTEGUI y TABERNA TOMPES, 1985: 143-144). Agradecemos la gentileza del organista e investigador zaragozano en facilitarnos este dato.

26 JAMBOU, 1988: $30\left(n^{\circ} 73\right)$ y $61\left(n^{\circ} 138\right)$. En su testamento y codicilio Francisco Gómez no alude a ellos, p. 15 (n³9 a y b).

27 JAMBOU, 1988: 19 ( $\mathrm{n}^{\circ} 43$ y 44), 35 ( $\mathrm{n}^{\circ} 86$ : en el inventario de Gaspar Brevos figuran "ocho moldes grandes y pequeños de hazer caños para órganos"), 41 ( $\mathrm{n}^{\circ} 102$ : el inventario de Andrés Gómez tiene varios formones") y $51\left(\mathrm{n}^{\circ} 114\right.$ : en este inventario de Jerónimo de Vargas, figuran moldes, grandes, pequeños o medianos). El vocablo "diapason" desaparece de los inventarios de los bienes de los organeros durante el siglo XVII y tampoco figura en los del XVIII en nuestro Evolución..., II, El inventario de Verdalonga (ibid., II, n“445, pp.248252), detallado, presenta un conjunto de herramientas del XVIII. Entre sus herramientas figura "una pantómetra" que se tasa en 8 reales (sobre este instrumento véase el valioso estudio de ROBLEDO ESTAIRE y BORDAS IBÁÑEZ, 1999: 215-313 [editado en inglés en Early Music, XXVII/3, 1998, con el título "José Zaragoza"box: science and music in Charles II"s Spain“]. Falta un estudio preciso lexicológico de éste y otros inventarios. El Diccionario Castellano con las voces de Ciencias y Artes de Terreros y Pandos (1786-1788) cuyos vocables de organería fueron escritos por el organero Fernández Dávila trae el vocablo "diapasón“ con su definición (JAMBOU, 1988, vol. II: 268).

\section{PRIMERAS AMPLIACIONES GEOGRÁFICAS}

El dibujo de 1584 de la catedral toledana es un valioso instumento de observación de la práctica diaria en la fábrica y afinación de los órganos y de los instrumentos de tecla. Herramienta pragmática el diseño representa un resultado de estudios teóricos anteriores o contemporáneos pero ha de ampliarse y sumarse a otros futuros hallazgos. En España los trabajos teóricos realizados por Goldaraz Gainza, A. García Pérez, Robledo/Bordas, M. Sanhuesa Fonseca, P. Otaola, A. Cea Galán o González Marín tendrán algún día su posible verificación y examen, su contraprueba en un número multiplicado, se puede esperar, de estos "diapasones".

John Koster nos invitó a volver a consultar y examinar los trabajos de Salomon de Caus ${ }^{28}$. El tratado Les Raisons des forces mouvantes avec diverses machines tant utilles que plaisants (Francfort-sur-le Main, $1615^{29}$ ) ofrece una representación de un diapasón que se funda aún en el Fefaut pero que llega al C5. Su vol. III representa así un dibujo que "vulgarmente dicho diapasón sirve para la medida de los caños de órganos", que se anuncia con el título "Comme il faut donner la mesure du Sisteme dit Diapason“. En 1584, Melchor de Miranda presentaba al cabildo toledano su «sistema» con estos dos vocablos: "medida“ y "diapasón" que ahora, en 1615/1624, se encuentran bajo la pluma de Salomon de Caus. Futuras investigaciones llegarán sin duda alguna a los capítulos que Marin Mersenne dedica al tema, citando a Salinas, y a la tabla que resume cuanto se ha de saber sin que "sea necesario a los organeros saber una cosa más“"30. Así se abre un camino en la organería, aspecto olvidado, por ingrato quizá, "quiero decir el tono, la entonación o diapasón de los órganos que conduce directamente al estudio metrológico o de las medidas, tema

28 Agradecemos al prof. John Koster (National Music Museum de Vermillion, Dakota del Sur, USA). No citamos aquí los trabajos consultados de Arnaud de Zwolle Les traités d'Henri-Arnaut de Zwolle..., Paris Picard, 1952.(Pl IX f 129). Llegado ya al pleno siglo XVII, se ha de observar que para la península -incluyendo a Portugal- falta aún un estudio sobre la difusión de las teorías musicales u organológicas que, pese al cambio politico cultural que sufre Europa a mediados del siglo XVII, parece se inclina en la peninsula más hacia Kircher que hacia Mersenne, lo que se nota hasta el siglo XIX por más que se haya estudiado la edición francesa de Dom Bedos de Celles. Ver Louis JAMBOU, 1987. de 1624

29 Hemos consultado (6/03/2014) en Gallica la edición de París

30 MERSENNE, 1636 (ed. 1986): 309, Libro “VI Des Orgues", 318 ss. En la página 339 en el capítulo XV "Expliquer toutes les Espèces de Diapasons,\& de Canons ou règles Harmoniques, dont on peut vser pour perfectionner les Orgues "viene la tabla a la cual alude el texto, 
añorado $\mathrm{ya}^{31}$ pero que ha de sedimentarse en épocas anteriores a los siglos XVIII o XIX» ${ }^{32}$.

\section{BIBLIOGRAFÍA}

BERMUDO, Juan, Declaración de instrumentos musicales, Osuna, 1555.

CALAHORRA MARTÍNEZ, Pedro, Música en Zaragoza. Siglos XVI-XVII, Zaragoza, Institución Fernando el Católico, 1977.

CAUX, Salomon de, Les Raisons des forces mouvantes avec diverses machines tant utilles que plaisants, Francfortsur-le Main, 1615, 1624.

DE GRAAF, Gerhard, El Órgano Español. Actas del I Congreso 27-29 octubre 1981 (Coord. Antonio Bonet Correa), Madrid, Universidad Complutense, 1984.

DE LA LAMA, S.J., Jesús Ángel, El órgano barroco español. I- Naturaleza, Valladolid, Junta de Castilla y León/Asociación Manuel Marín, 1995.

ESTEVES PEREIRA, Luis Artur (ed.), Tratado $2^{\circ}$ de geometría prática, Lisboa, Fundación Calouste Gulbenkian, 1984.

FERNÁNDEZ COLLADO, Ángel, RODRÍGUEZ GONZÁLEZ, Alfredo, CASTAÑEDA TORDERA, Isidoro, Los diseños de la catedral de Toledo. Catálogo de diseños arquitectónicos, artísticos, topográficos, $y$ textiles, Toledo, Instituto Teológico San Ildefonso Colección Primatialis Ecclesias Toletanae Memoria, 5, 2009.

JAMBOU, Louis (ed.), Compendio del Arte de Organería, Madrid, SEdeM, 1987.

JAMBOU, Louis, Evolucón del órgano español. Siglos XVI-XVIII. Oviedo, Universidad, Publicaciones, col. Ethos-Música, 1988.

JAMBOU, Louis, "Algunas consideraciones en torno a modelos teóricos musicales y prácticas instrumentales en el siglo XVI“" en Cinco siglos de música de tecla española (ed. Luisa Morales), Garrucha (Almería), Asociación Cultural Leal, 2007: 125-136.

MERSENNE, Marin, Harmonie universelle, Paris, 1636 (ed. François Lesure, Paris, CNRS, 1986).

ROBLEDO ESTAIRE, Luis y BORDAS IBÁÑEZ, Cristina, "El arcón de José Zaragoza: ciencia y música en la España de Carlos II“, en Nassarre, XV/1-2 (1999): 215-313.

SAGASETA ARIZTEGUI, Aurelio y TABERNA TOMPES, Luis, Órganos de Navarra, Pamplona, Institución Príncipe de Viana, 1985.

SAURA BUIL, Joaquín, Diccionario técnico-histórico del Órgano en España, Barcelona, CSIC, 2001.

31 Alude a ello, pensando sobre todo en el XIX, Gerhard DE GRAAF, 1984: 371 .

32 El 3/12/2011 pronuncié una conferencia en el marco del Congreso del Órgano Español. Su título era: "Investigar en el campo de la organeria: balance de una trayectoria" y terminaba con estas reflexiones. 


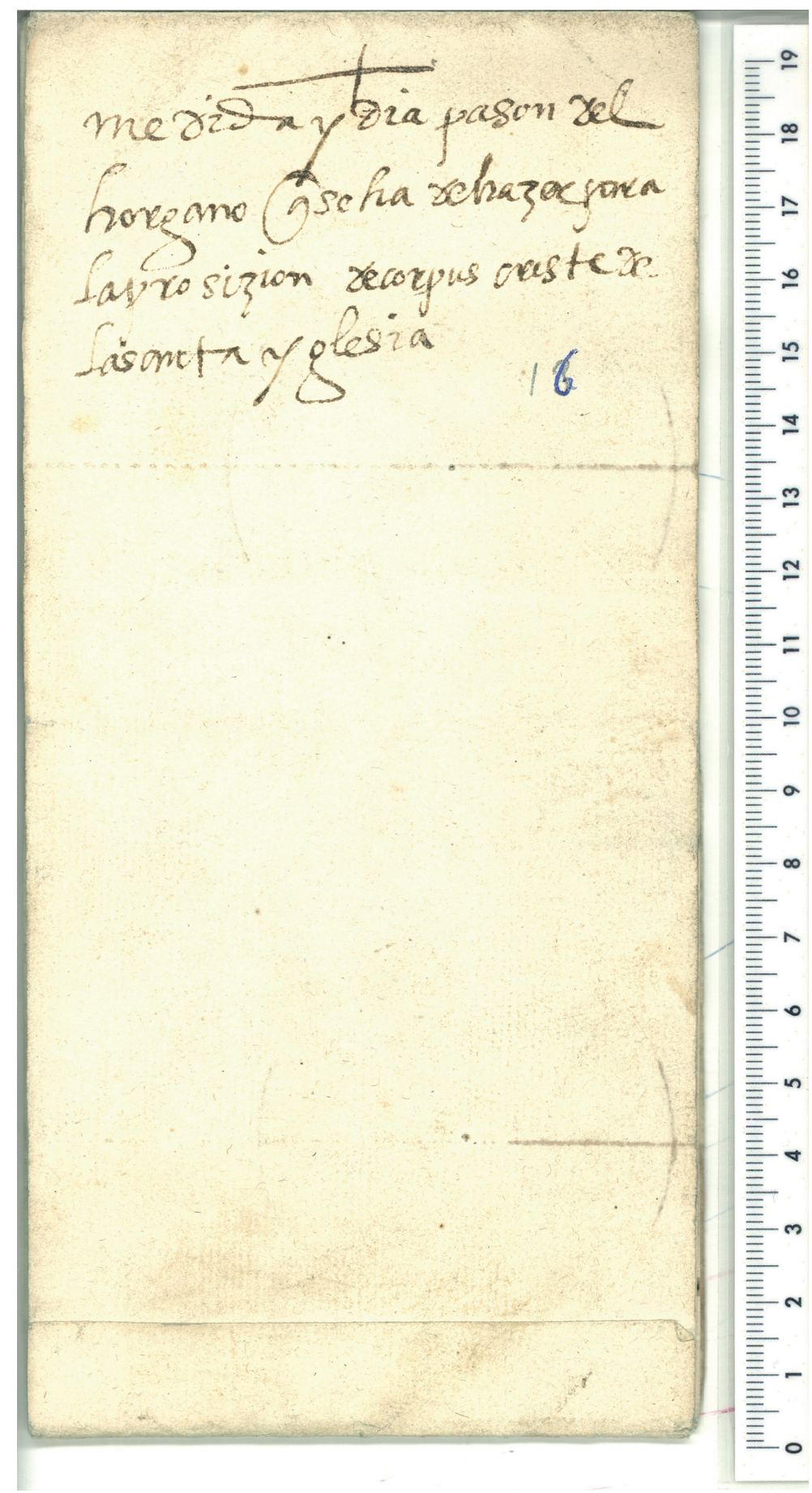




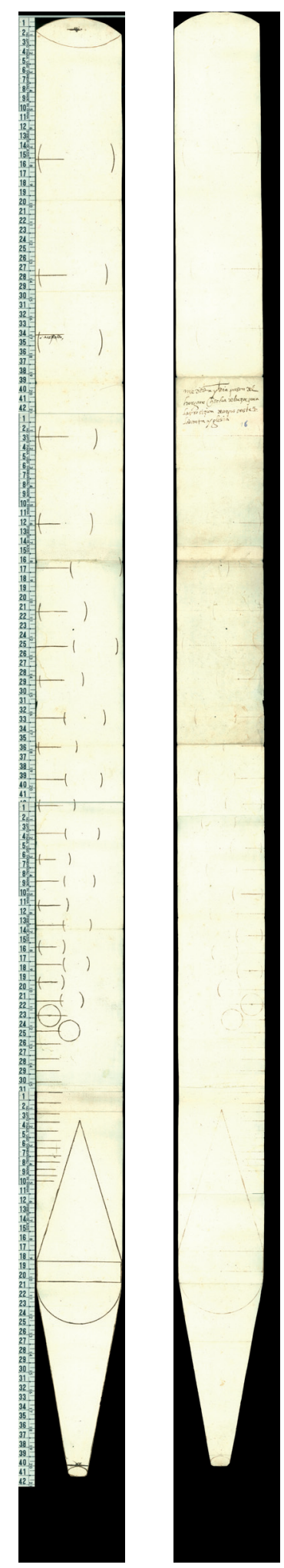

